\title{
Generation of Boundary Manikin Anthropometry
}

Karen S. Young

Sarah Margerum

Abbe Barr

Lockheed Martin Mission Services

Mike A. Ferrer

MEI Technologies Inc

Sudhakar Rajulu, Ph.D.

NASA, Johnson Space Center

\section{ABSTRACT}

The purpose of this study was to develop 3D digital 'boundary manikins' that are representative of the anthropometry of a unique population. These digital manikins can be used by designers to verify and validate that the components of the spacesuit design satisfy the requirements specified in the Human Systems Integration Requirements (HSIR) document. Currently, the HSIR requires the suit to accommodate the $1^{\text {st }}$ percentile American female to the $99^{\text {th }}$ percentile American male.

The manikin anthropometry was derived using two methods: Principal Component Analysis (PCA) and Whole Body Posture Based Analysis (WBPBA). PCA is a statistical method for reducing a multidimensional data set by using eigenvectors and eigenvalues. The goal is to create a reduced data set that encapsulates the majority of the variation in the population. WBPBA is a multivariate analytical approach that was developed by the Anthropometry and Biomechanics Facility (ABF) to identify the extremes of the population for a given body posture. WBPBA is a simulation-based method that finds extremes in a population based on anthropometry and posture whereas PCA is based solely on anthropometry.

Both methods yield a list of subjects and their anthropometry from the target population; PCA resulted in 20 female and 22 male subjects' anthropometry and WBPBA resulted in 7 subjects' anthropometry representing the extreme subjects in the target population. The subjects' anthropometry is then used to 'morph' a baseline digital scan of a person with the same body type to create a 3D digital model that can be used as a tool for designers, the details of which will be discussed in subsequent papers.

\section{INTRODUCTION}

The Constellation Program is tasked to adhere to the requirements specified in the Human Systems Integration Requirement (HSIR) document [1] for the design of the future space suits. The current design requirements state that the suit must accommodate a $1^{\text {st }}$ percentile female to a $99^{\text {th }}$ percentile male for a set of specific critical dimensions. To assist in the design as well as verify the requirements have been met, a methodology was developed to provide realistic three dimensional models covering the entire range of critical dimensions within the population. The digital manikins act as templates on which the computer aided design (CAD) suit models can then be overlaid to verify that the suit design accommodates the required range of anthropometric sizes. These boundary manikins contain realistic anthropometry, with different combinations of lengths, widths, and circumference for the body segments. This variety provides a realistic assortment of body shapes for a designer to allocate the number of suit components that ensure accommodation levels specified by the HSIR requirements.

PRINCIPAL COMPONENT ANALYSIS (PCA) - Often times a system is defined by more than three variables or dimensions. Trying to represent a four-dimensional data set visually is extremely abstract. PCA is a method to reduce a multi-dimensional dataset to a more manageable size [2]. The dataset used in this PCA were relative to space suit design and were reduced to three orthogonal components comprised of a linear 
combination of the original body dimensions [2]. The eigenvectors and eigenvalues are calculated by transforming a multi-dimensional dataset of correlated variables into a smaller set of variables that account for the greatest amount of variability within the dataset, or principal components. For example, if a dataset has 10 variables the PCA will return 10 principal components, consisting of 10 eigenvectors and 10 eigenvalues. However, the first eigenvector, or principal component, accounts for the maximum amount of variability in the dataset and each of the subsequent principal components account for the remainder of the variability in descending order [3]. The eigenvalues represent the magnitude of the variability in the principal component. Typically, only a select few principal components are selected to represent the dataset, the eigenvalues for each of these components are summed to determine the percent variance of all the selected eigenvectors; ultimately a high percent variability is desired to create a more accurate result. Only the first few principal components represent the major axes of variation, usually two to three eigenvectors and eigenvalues, while the others remaining represent minimal variation and can be discarded and not included in the summation of the eigenvalues to determine the percent variability accommodated [4].

The selected principal components are then mapped in principal component space to determine the feature points that will be used to generate the boundary manikin anthropometry.

WHOLE BODY POSTURE BASED ANALYSIS (WBPBA) - The Whole-Body Posture Based Analysis (WBPBA) is a methodology previously developed by the $A B F$ for identifying the extremes of the population for a given body posture using a multivariate analytical approach [5]. Unlike the Principal Component Analysis which provides information of subjects spanning a range of percentiles for each dimension, the WBPBA provides anthropometries on a selected percentile value, for this study the extremes were selected, $1^{\text {st }}$ percentile and $99^{\text {th }}$ percentile for a subset of measurements.

To perform WBPBA a functional pose is chosen for analysis. From this functional posture a unique set of anthropometric parameters are identified from the sagittal, transverse, and frontal plane views. The square root of the sum of the squares is then calculated to determine a set of worse-case anthropometries which then become a set of boundary manikins.

\section{METHODS}

PCA - A custom written MATLAB (The MathWorks Inc. Natick, Ma) program by the $A B F$ was developed to analyze the HSIR database [1]. The custom MATLAB program allows the user to perform a PCA in either 2D or 3D space and for either male or female datasets.
A parsed data set from the HSIR database was used for PCA. The HSIR database, which consists of 125 measurements, was reduced to the suit specific measurements listed in the HSIR document [1] and Soyuz TMA measurements for PCA (Table 1). The reduced dataset was then standardized. The method of standardization used for PCA was to divide the dataset by the standard deviation for each dimension. The dataset was then further truncated by eliminating those subjects from the dataset that exceed the range of $1^{\text {st }}$ to $99^{\text {th }}$ percentile for each dimension.

Table 1: Suit Specific and Soyuz TMA Measurements

\begin{tabular}{|c|c|}
\hline $\begin{array}{l}\text { HSIR Suit Specific } \\
\text { Measurements }\end{array}$ & $\begin{array}{c}\text { Soyuz TMA } \\
\text { Measurements }\end{array}$ \\
\hline $\begin{array}{c}\text { Stature } \\
\text { Crotch Height } \\
\text { Chest Breadth } \\
\text { Chest Depth } \\
\text { Chest Circumference } \\
\text { Head Breadth } \\
\text { Head Length } \\
\text { Thigh Circumference } \\
\text { Bicep Circumference, Flexec } \\
\text { Abdominal Extension (Waist } \\
\text { Depth) } \\
\text { Hip Breadth } \\
\text { Weight* } \\
\text { Wall to Wrist } \\
\text { Inter Wrist } \\
\text { Inter Elbow } \\
\text { Knee Height, Mid-Patella } \\
\text { Vertical Trunk Diameter }\end{array}$ & $\begin{array}{c}\text { Soyuz Sitting Height* } \\
\text { Foot Length } \\
\text { Inter Scye Width } \\
\text { Stature } \\
\text { Weight* } \\
\text { Hip Breadth } \\
\text { Bi-Deltoid Breadth* } \\
\text { Thigh to Thigh*}\end{array}$ \\
\hline
\end{tabular}

The prepared dataset was then imported into the custom $A B F$ written MATLAB program which performed the PCA. The outputs of this function were the eigenvectors, eigenvalues, and the dataset plotted in PCA space.

Three principal components (eigenvectors) were selected from the PCA results; subsequently a percent variability of $69 \%$ for the female dataset and $68 \%$ variability for the male dataset were determined from the summed eigenvalues for those three eigenvectors (Table 2 and Figure 1).

\begin{tabular}{|c|c|c|}
\hline Component & Eigenvalue (\%) & Cumulative (\%) \\
\hline PC 1 & 36.7 & 36.7 \\
\hline PC 2 & 22.4 & 59.1 \\
\hline PC 3 & 10.3 & 69.4 \\
\hline
\end{tabular}




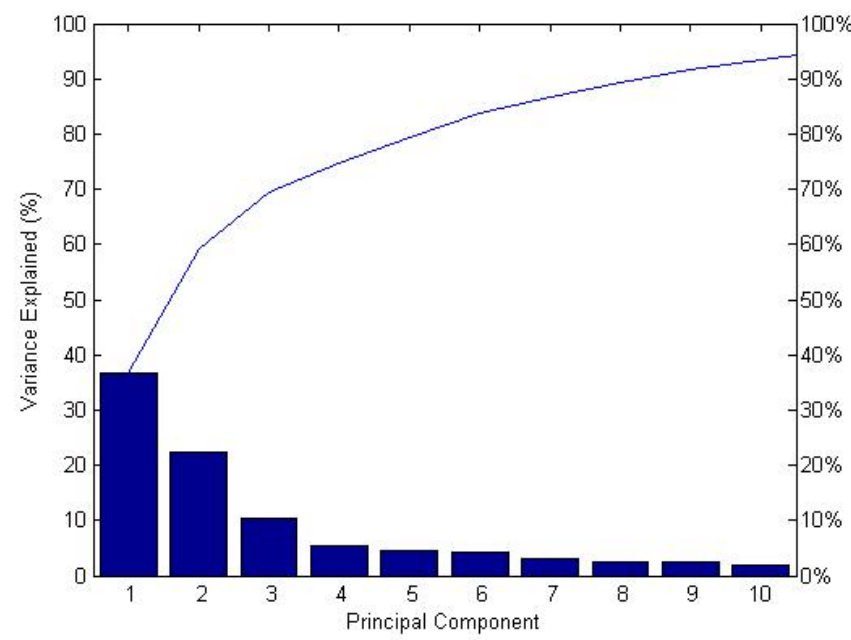

Figure 1: Female Percent Variability Accommodation: The bars are percent variability for each principal component and the line displays the summation of variability as the number of components increase

After the three principal components were determined the results were plotted in PCA space, with each data point representing a subject's anthropometry represented in eigenvectors. The population forms an ellipsoid within a three dimensional PCA space, if only two principal components were selected this representation would become simply an ellipse. The subjects closer to the center of the ellipsoid would represent the average subjects whereas the subjects close to the edge of the ellipsoid represent the boundary manikins. The boundary manikins were preferred because they would have a dimension close to the extreme of the population. For example, if the 1st principal component represents limb length (arm and leg lengths), the 2nd principal component represents torso length, and the $3^{\text {rd }}$ principal component represents body girth, those subjects in the center of the ellipse would represent subjects of average limb length, average torso length, and average girth. Those subjects at the extremes of the 1st principal component would represent subjects of average torso length and average girth that have either long or short arm and leg lengths. The extremes of the 2nd principal component would represent subjects of average limb lengths, average body girth, and small or large torso lengths. The extremes of the $3^{\text {rd }}$ principal component would represent subjects of average limb lengths, average torso length, and small or large body girth (skinny or stout).

To determine which subjects could be considered as boundary manikins from the PCA analysis, the appropriate accommodation ellipsoid needed to be created with the center representing the mean of the PCA axes. In this study a 99\% accommodation ellipsoid was used as the accommodation boundary to adhere to the HSIR requirements. This was created using three standard deviations of each principal component or axis to obtain the radii for the ellipsoid. Next, points along the surface of the ellipsoid were identified to represent the extreme individuals; these points were called feature points. For a three dimensional PCA space the feature points were found at the midpoints of each quadrant, axis intercepts, and four points in each plane (Table 3 ).

Table 3 Feature Points for 3D Ellipsoid

\begin{tabular}{|c|c|}
\hline \multicolumn{2}{|c|}{ Points along each Plane } \\
\hline$(+, 0,-)$ & $\mathrm{XZ}$ Plane, $\mathrm{Y}=0$ \\
\hline$(-, 0,-)$ & $X Z$ Plane, $Y=0$ \\
\hline$(-, 0,+)$ & $\mathrm{XZ}$ Plane, $\mathrm{Y}=0$ \\
\hline$(+, 0,+)$ & $X Z$ Plane, $Y=0$ \\
\hline$(0,+,-)$ & YZ Plane, $\mathrm{X}=0$ \\
\hline$(0,+,+)$ & YZ Plane, $\mathrm{X}=0$ \\
\hline$(0,-,-)$ & YZ Plane, $\mathrm{X}=0$ \\
\hline$(0,-,+)$ & YZ Plane, $X=0$ \\
\hline$(+,+, 0)$ & XY Plane, $Z=0$ \\
\hline$(-,+, 0)$ & XY Plane, $Z=0$ \\
\hline$(-,-, 0)$ & XY Plane, $Z=0$ \\
\hline$(+,-, 0)$ & XY Plane, Z=0 \\
\hline \multicolumn{2}{|c|}{ Mid Quadrant Points } \\
\hline$(+,+,+)$ & Quadrant $1,+Z$ \\
\hline$(-,+,+)$ & Quadrant 2, +Z \\
\hline$(-,-,+)$ & Quadrant 3, +Z \\
\hline$(+,-,+)$ & Quadrant $4,+Z$ \\
\hline$(+,+,-)$ & Quadrant 1, -Z \\
\hline$(-,+,-)$ & Quadrant 2, -Z \\
\hline$(-,-,-)$ & Quadrant 3, -Z \\
\hline$(+,-,-)$ & Quadrant 4, -Z \\
\hline \multicolumn{2}{|c|}{ Axis Intercept Points } \\
\hline$(+, 0,0)$ & Positive $X$ axis intercept \\
\hline$(0,+, 0)$ & Positive $Y$ axis intercept \\
\hline$(-, 0,0)$ & $\begin{array}{l}\text { Negative } X \text { axis } \\
\text { intercept }\end{array}$ \\
\hline$(0,-, 0)$ & $\begin{array}{l}\text { Negative } Y \text { axis } \\
\text { intercept }\end{array}$ \\
\hline$(0,0,+)$ & Positive $\mathrm{Z}$ axis intercept \\
\hline$(0,0,-)$ & $\begin{array}{l}\text { Negative } Z \text { axis } \\
\text { intercept }\end{array}$ \\
\hline
\end{tabular}

From the feature points the boundary manikins' anthropometry were identified by selecting the subject that was closest to the feature point within the ellipsoid, termed the nearest neighbor. To find the nearest neighbor(s) to the feature points the distance formula was used (Equation 1) and the subject with the smallest distance to the feature point was chosen as the nearest 
neighbor. This resulted in 26 manikin anthropometries for each gender, corresponding to each feature point in the PCA space. The program was also developed with the capability of finding the five closest neighbors. This yields potentially five times as many boundary manikins. However, only one nearest neighbor was used for this analysis (Figure $2 \& 3$ ).

$$
\text { Distance }=\sqrt{\left(\left(\mathrm{x}_{2}-\mathrm{x}_{1}\right)^{2}+\left(\mathrm{y}_{2}-\mathrm{y}_{1}\right)^{2}+\left(\mathrm{z}_{2}-\mathrm{z}_{1}\right)^{2}\right)}
$$

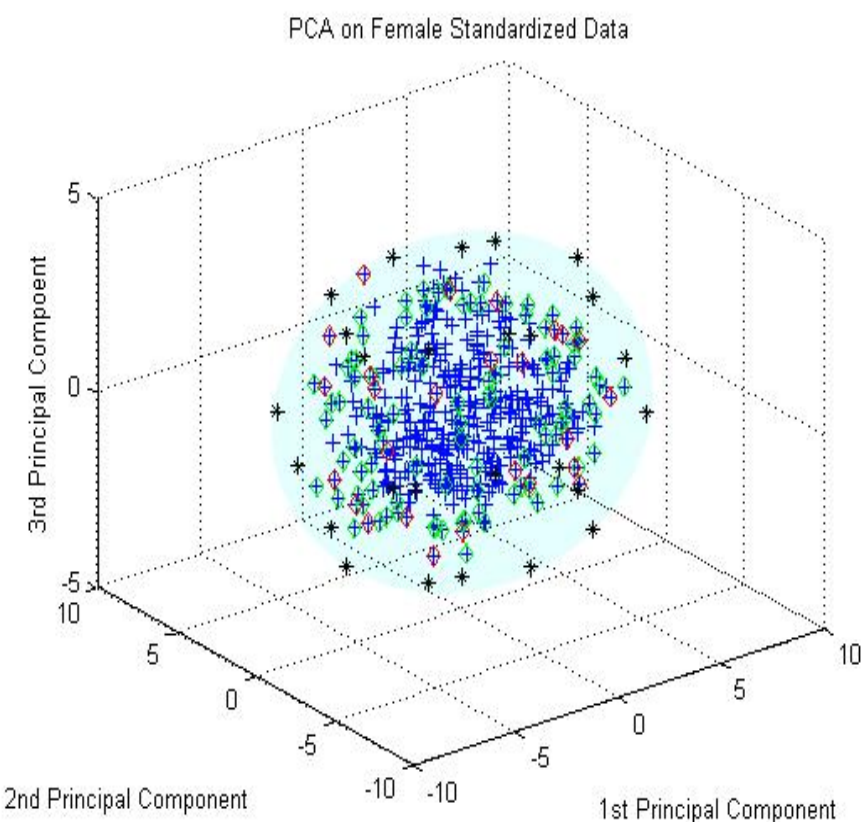

Figure 2: Female PCA in 3D. The black asterisks represent the feature points; the red diamonds represent the nearest neighbor with the minimum distance to the feature point. The green diamonds represent the next four closest neighbors to the feature point

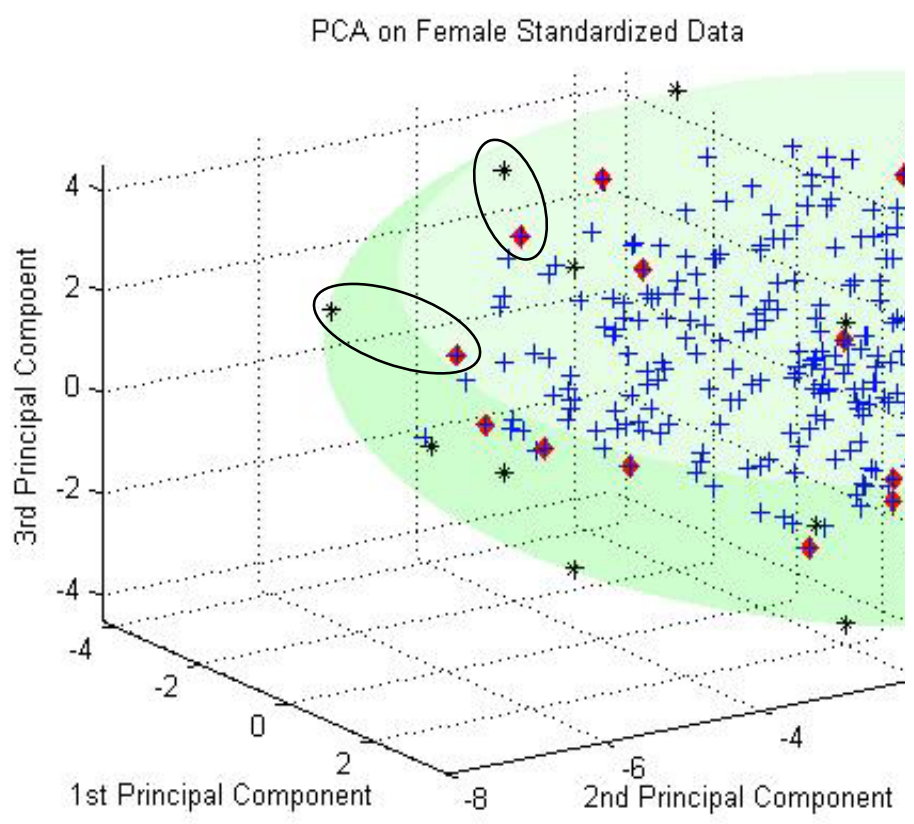

Figure 3: Female PCA in 3D. Circled areas are two examples of the feature points and the corresponding nearest neighbor.

WBPBA - As with PCA, a custom written MATLAB program was created by the $A B F$ as a tool to analyze the HSIR database [1]. The WBPBA MATLAB program was developed to allow the user to perform a WBPBA on either male or female datasets for $99^{\text {th }}$ percentile or $1^{\text {st }}$ percentile values.

A parsed data set from the HSIR database was also used for this analysis. The HSIR database was reduced to measurements that were specific to a T-pose posture (Table 4). The T-pose posture was chosen for this analysis since it is one of the primary postures used during a suit sizing fit check to make sure that there is enough length in the arms and that the subject's shoulders are in the middle of scye bearing. Measurements for the T-pose were determined by examining this posture from the frontal, transverse, and sagittal planes of the body.

Table 4: WBPBA Measurements for T-pose

\begin{tabular}{cc} 
& Measurements \\
\hline 1 & Stature \\
2 & Crotch Height \\
3 & Arm Span \\
4 & Hip Breadth \\
5 & Lateral Femoral Height \\
6 & Vertical Trunk Circumference \\
7 & Head Circumference \\
8 & Chest Circumference \\
9 & Waist Circumference \\
10 & Elbow Circumference \\
11 & Thigh Circumference \\
\hline
\end{tabular}


Once the dimensions to be used in the analysis were determined, the dataset was then normalized to minimize the impact any large valued measurements would have on the results. The normalization method used was to calculate the z-scores for each subject in the dataset, for each dimension. The z-scores were calculated by subtracting the average value from each individual data point for a given measurement and divide the quantity by the standard deviation of the given measurement (Equation 2).

$$
\text { Zscore }_{\mathrm{i}}=\left(\mathrm{X}_{\mathrm{i}}-\mathrm{X}_{\text {Average }}\right) / \sigma
$$

Equation 2

After the dataset had been reduced and normalized, the final step was to truncate the number of subjects by eliminating those subjects less than $1^{\text {st }}$ percentile female and greater than $99^{\text {th }}$ percentile male in each of the critical dimensions. This step was performed to adhere to requirements set forth in the HSIR that accommodation levels must range from a $1^{\text {st }}$ percentile female to a $99^{\text {th }}$ percentile male [1].

The prepared dataset was then imported into the $A B F$ custom written MATLAB program. For this analysis the WBPBA was performed on both the normalized and nonnormalized datasets to compare the impact of larger measurements on the results. Using the $1^{\text {st }}$ percentile and $99^{\text {th }}$ percentile vales for each measurement, the dataset(s) were then sorted from least to greatest and the five neighbors nearest to the $99^{\text {th }}$ percentile value (or $1^{\text {st }}$ percentile) were determined for each measurement (Table 5). The five nearest neighbors were selected in order to provide a larger dataset to work with and to ultimately select the most representative extreme subject. The end result was a large matrix consisting of the 5 closest neighbors to the $99^{\text {th }}$ percentile for Measurement 1 (Table 4), the 5 closest subjects to the $99^{\text {th }}$ percentile for Measurement 2 (Table 4), and so on until a total of 55 subjects were harvested from the database corresponding to the 5 largest for each of the eleven selected measurements. This was repeated for the $1^{\text {st }}$ percentile condition.

With this subset of 55 subjects across the eleven (n) measurements (Measurement ${ }_{n}$ ), the square root of the sum of squares (SRSS) was determined for each subject (Equation 3). The maximal value (minimal for $1^{\text {st }}$ percentile calculations) for each group of 5 nearest neighbors was selected and designated as a boundary manikin. The end result of the analysis was 11 manikins corresponding to each body measurement of interest (Table 5). This process was repeated for each combination of male/female, $1^{\text {st }} / 99^{\text {th }}$, and normalized/non-normalized configurations yielding a total of eighty-eight manikins.

$$
\text { SRSS }=\sqrt{\sum_{i=1}^{n} \text { Measurement }_{i}{ }^{2}}
$$

Equation 3

Table 5: Example of reduced results from a male, non-normalized, 99th percentile WBPBA

\begin{tabular}{|l|l|l|l|l|l|}
\hline & $\begin{array}{l}\text { Stature } \\
(\mathbf{m m})\end{array}$ & $\cdots$ & $\begin{array}{l}\text { Waist } \\
\text { Circ } \\
(\mathbf{m m})\end{array}$ & SRSS & $\begin{array}{l}\text { Subject } \\
\#\end{array}$ \\
\hline $\begin{array}{l}\mathbf{9 9}^{\text {th }} \text { \% } \\
\text { value }\end{array}$ & 1945 & & 1106 & & \\
\hline $\begin{array}{l}\mathbf{1}^{\text {st }} \\
\text { value }\end{array}$ & 1626 & & 696 & & \\
\hline Manikin & & & & & \\
\hline 1 & 1921 & & 1005 & 3941 & 21904 \\
\hline 2 & 1907 & & 1041 & 3879 & 15896 \\
\hline 3 & 1921 & & 1005 & 3941 & 21904 \\
\hline 4 & 1899 & & 1045 & 3911 & 3771 \\
\hline 5 & 1903 & & 938 & 3888 & 2785 \\
\hline 6 & 1921 & & 1005 & 3941 & 21904 \\
\hline 7 & 1838 & & 1089 & 3877 & 6342 \\
\hline 8 & 1892 & & 1004 & 3889 & 23480 \\
\hline 9 & 1907 & & 1041 & 3879 & 15896 \\
\hline 10 & 1899 & & 1045 & 3911 & 3771 \\
\hline 11 & 1838 & & 1089 & 3877 & 6342 \\
\hline & & & & & \\
\hline Max & 1921 & & 1089 & 3941 & \\
\hline Min & 1838 & & 938 & 3877 & \\
\hline
\end{tabular}

\section{RESULTS}

PCA - The 26 boundary manikins for each gender were then converted from PCA space values back into the original anthropometry (consisting of 125 measurements), using the subject numbers as indices. With over 52 manikins in total, it became necessary to reduce the final list to a concise representative sample that still spanned the range of critical anthropometries. There were several different attempts to try to reduce the number of manikins that resulted from the 3D PCA analysis. The vast amount of manikins proved difficult to differentiate and compare various attributes. In order to ensure that the manikins represented the full spectrum of each measurement, the 26 manikins' dimensions were converted to percentiles and plotted on a graph that contained all measurements of interest sequentially (Figure 4). In the initial look at the data from $1^{\text {st }}$ to $99^{\text {th }}$ percentile for each dimension, it was noted that there was adequate coverage and a sufficient spread for all dimensions for both genders (Figure 4). The method employed in reducing the dataset involved observing the large gaps present in the data, and identifying the manikins at the end points of each gap. These endpoint manikins were classified as automatic "keepers" in order to ensure the overall distribution of the measurements was maintained. 
To determine the initial "keeper" manikins an optimization method was used to determine how large of a gap width was needed to identify a select number of manikins while sustaining a sufficient spread of the data. The remaining subset of manikins was then compared against the keeper manikins' percentile values across all measurements (blue data points in Figure 4). An individual manikin was displayed (red circles in Figure 4) to show where it fell within each given measurement. Each individual manikin was reviewed to determine if it could be discarded without affecting the overall spread of the data. Key gaps were identified where the loss of the remaining boundary manikins would be detrimental to the overall analysis. If no critical regions were identified and there was no substantial impact on removing a manikin from the pool, the manikin could be safely discarded. This reduction method resulted in a final anthropometric dataset consisting of 20 female and 22 male manikins.

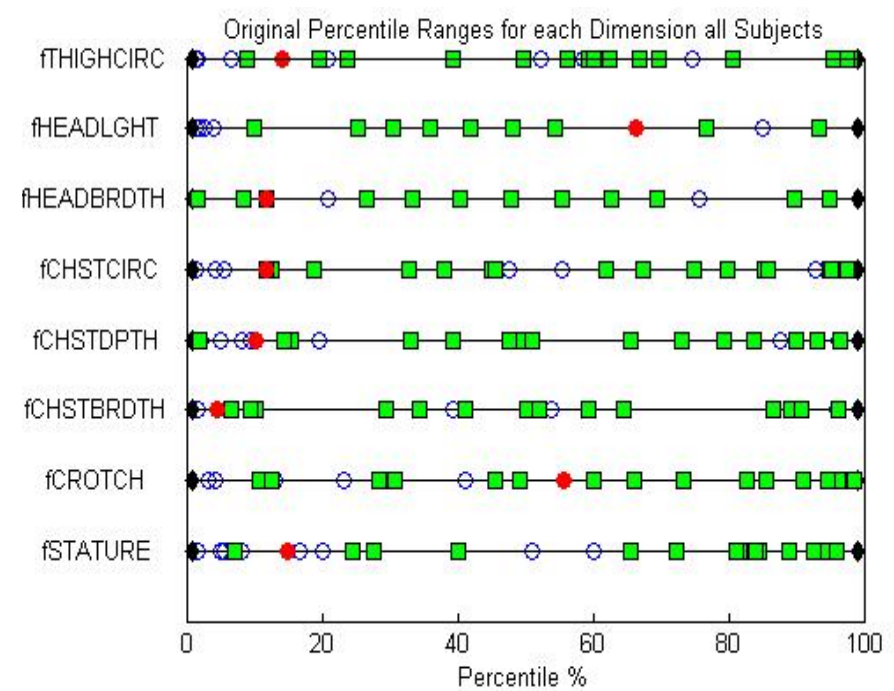

Figure 4: Example plot of manikin percentile values for each dimension. Green squares represent "keeper" manikins, blue circles represent possible manikins for reduction, and the red circles represent the specific manikin for analysis. Not all critical measurements are shown.

WBPBA - The WBPBA resulted in eighty-eight manikins: eleven from each combination of male and female, $1^{\text {st }}$ and $99^{\text {th }}$ percentile, normalized and un-normalized datasets. In order to reduce the number to a workable size, it was ultimately decided to take the manikins who had the maximal/minimal overall SRSS value from each configuration, basically the largest, or smallest in the case of the $1^{\text {st }}$ percentile, SRSS values for each group of nearest neighbors. Therefore the manikins with the maximal $\left(99^{\text {th }}\right.$ percentile WBPBA $)$ or minimal $\left(1^{\text {st }}\right.$ percentile WBPBA) square root of the sum of squares (SRSS) values were determined for normalized and nonnormalized datasets, resulting in eight manikins.

When comparing the normalized vs. un-normalized results there was one manikin that was designated a boundary manikin in both cases, creating a redundancy that resulted in 7 unique manikins when the duplicate was removed. Of the resulting 7 manikins one was also deemed a boundary manikin in the PCA analysis. With the duplicates removed from both analyses the WBPBA and PCA yielded a total of 48 manikins based on real subjects' anthropometries.

\section{CONCLUSION}

The ABF successfully developed two analytical tools to identify and generate boundary manikin anthropometries that represent realistically proportioned body shapes and sizes. Representing the vast differences in body shapes was of highest importance in order to capture the variation in the sample population that uses the space suit systems to safely and efficiently perform their tasks. Upon successful reduction techniques the number of manikins was reduced from 138 to 48 manikins, realistically capturing the multitude of body morphologies potentially interfacing the future space suit system while maintaining the integrity and distribution of the data within the requirements specified in HSIR (Table 6).

Table 6: PCA \& WBPBA manikin ranges $(\mathrm{cm})$ and HSIR ranges $(\mathrm{cm})$

\begin{tabular}{|c|c|c|c|c|}
\hline \multirow[b]{2}{*}{ Dimension } & \multicolumn{2}{|c|}{$\begin{array}{l}\text { Multivariate } \\
\text { Range (cm) }\end{array}$} & \multicolumn{2}{|c|}{ HSIR range $(\mathrm{cm})$} \\
\hline & Min & $\operatorname{Max}$ & Min & $\operatorname{Max}$ \\
\hline Stature & 150.1 & 192.1 & 148.7 & 194.5 \\
\hline Crotch Height & 68.6 & 95.7 & 66.5 & 95.8 \\
\hline Chest Breadth & 25.2 & $39.9^{*}$ & 23.5 & 39.3 \\
\hline Chest Depth & 19.3 & 29.6 & 19.1 & 30.3 \\
\hline Chest Circ & 76.9 & 117.6 & 75.7 & 118.6 \\
\hline Head Breadth & 13.4 & 16.4 & 13.3 & 16.6 \\
\hline Head Length & 17.4 & 20.7 & 17.2 & 21.5 \\
\hline $\begin{array}{l}\text { Thigh Circ } \\
\text { Bicep Circ }\end{array}$ & 48.3 & 70.5 & 47.7 & 71.8 \\
\hline $\begin{array}{l}\text { Flexed } \\
\text { Abdominal }\end{array}$ & 23.0 & 39.3 & 22.9 & 40.4 \\
\hline Extension & 16.4 & 28.0 & 16.9 & 30.1 \\
\hline Hip Breadth & 30.7 & 39.8 & 29.8 & 40.6 \\
\hline Wall to Wrist & 55.5 & 77.3 & 54.7 & 77.6 \\
\hline Inter Wrist & 117.5 & 160.8 & 115.1 & 161.8 \\
\hline Inter Elbow & 73.9 & 101.8 & 72.5 & 101.5 \\
\hline $\begin{array}{l}\text { Knee Height, } \\
\text { mid patella } \\
\text { Vertical Trunk }\end{array}$ & 40.8 & 56.9 & 39.6 & 57.8 \\
\hline Diameter & 58.3 & 75.6 & 56.3 & 76.3 \\
\hline Sitting Height & 79.0 & 98.5 & 77.6 & 101.3 \\
\hline Foot Length & 23.1 & 29.9 & 21.5 & 30.5 \\
\hline Interscye Width & 31.2 & 47.2 & 29.3 & 48.1 \\
\hline
\end{tabular}

Principal Component Analysis can be applied to any set of anthropometry tailored to a specific analysis and the relevant boundary manikin anthropometry can be identified. PCA was used as a statistical method to obtain subject anthropometries that represent the extreme body sizes and shapes that are realistically proportioned. These manikins were verified to be 
sufficient in percent variability accommodation levels representing the entire range of $1^{\text {st }}$ to $99^{\text {th }}$ as stated in the HSIR requirements. This method resulted in a total of 20 female and 22 male boundary manikins after data reduction techniques were employed.

The ABF developed WBPBA can be applied to any functional posture, utilizing measurements from all three planes, transverse, sagittal, and frontal to identify worse case anthropometries. WBPBA can be used to automate the methodology of parsing through the data and generating manikin values using the HSIR database based on posture.

Both of these analytical tools provided a set of anthropometric data from which baseline 3D whole body scans of the same body type can be 'morphed' to achieve 3D models with the anthropometry of the boundary manikins (to be discussed in subsequent work). The boundary manikin models were created as a tool for designers of the next generation space suit to verify that their models stay within the requirements specified in HSIR. Ultimately, the future uses of these tools will have critical value to the space program by providing the ability to identify critical representative anthropometries for a given body posture and to generate a viable manikin with applications as a development tool in the design process.

One limitation to the analyses is the selection of the dimensions used in both PCA and WBPBA. In PCA, measurements specific to suit design and the Soyuz TMA seat liner requirements were used. WBPBA used dimensions applicable to a T-posture. However there were several other measurements that could have been included or discarded to change the number of measurements used in these analyses. Modification to these measurements will affect the number of manikins and their body sizes and shapes of the final manikins. As with any statistical analysis the dimensions used should be relevant to the design.

Another limitation was the optimization method used in reducing the number of manikins. A robust methodology was administered to reach the final number of manikins from these analyses. Special care was taken to ensure that the manikins maintained a suitable representation of the target population from the 1st to 99th percentile range. Further research in other modes of optimization may be focused on to automate the reduction process.

The $A B F$ is working to determine if there are additional boundary manikins that may provide greater insight to the designer. These may include manikins with unique anthropometry combinations or extreme manikin anthropometries for certain operations. The ABF will support the generation of these boundary manikins and provide a useful comprehensive data set to the suit designers.

\section{ACKNOWLEDGMENTS}

The authors would like to thank the XA Project Office at Johnson Space Center for their support and funding of this research.

\section{REFERENCES}

[1] CxP 70024, Human-Systems Integration Requirements (HSIR) (C000114)

[2] Gordon, C. "Mulitvariate Anthropometric Models for Seated Workstation Design", U.S. Army Natick Soldier Center, Natick, MA

[3] 'Principal Component Analysis', (c) djmw, 3/23/1999 http://www.fon.hum.uva.nl/praat/manual/Principal c omponent analysis.html

[4] Zehner, Gregory, "Prediction of Anthropometric Accommodation in Aircraft Cockpits", Ohio State University, Dissertation, Dayton, $\mathrm{OH}, 2000$

[5] Gonzalez, L.J., Rajulu, S.L. (2003). "Posture-Based Whole Body Anthropometric Analysis - A Case Study", Digital Human Modeling For Design And Engineering Conference And Exhibition, June 2003, Montreal, Canada.

\section{CONTACT}

Karen Young

Lockheed Martin

Karen.S.Young@nasa.gov

Sudhakar Rajulu

NASA, Johnson Space Center

Sudhakar.Rajulu-1@nasa.gov 